\title{
A genome screen for multiple sclerosis in Sardinian multiplex families
}

\author{
Francesca Coraddu ${ }^{1}$, Stephen Sawcer ${ }^{1}$, Sandra D’Alfonso ${ }^{2}$, Marina Lai ${ }^{3}$, Anke Hensiek ${ }^{1}$, \\ Elisabetta Solla ${ }^{3}$, Simon Broadley ${ }^{1}$, Cristina Mancosu ${ }^{3}$, Maura Pugliatti ${ }^{4}$, \\ Maria Giovanna Marrosu*,3 and Alastair Compston ${ }^{1}$
}

${ }^{1}$ University of Cambridge Neurology Unit, Addenbrooke's Hospital, Hills Road, Cambridge, CB2 2QQ UK;
${ }^{2}$ Department of Medical Sciences, University of Piemonte Orientale 'A. Avogadro', Novara, Italy; ${ }^{3}$ Centro per la
diagnosi e cura per la Sclerosi Multipla, Universita' di Cagliari, ASL8 Ospedale Binaghi, via Is Guaddazonis,
09100 Cagliari, Italy; ${ }^{4}$ Clinica Neurologica dell'Universita' di Sassari. Viale San Pietro Sassari, Italy

The prevalence of multiple sclerosis in Sardinia is significantly higher than in neighbouring Mediterranean countries, suggesting that the isolated growth of the population has concentrated genetic factors which increase susceptibility to the disease. The distinct HLA association of multiple sclerosis in Sardinia supports this interpretation. We have performed a whole genome screen for linkage in 49 Sardinian multiplex families using 327 markers. Non parametric linkage analysis of these data reveal suggestive linkage in the region of Chr 1q31, Chr 10q23 and Chr 11p15. European Journal of Human Genetics (2001) 9, 621-626.

Keywords: multiple sclerosis; linkage; genome screens; Sardinians

\section{Introduction}

To date, four whole genome screens for linkage have been completed in multiple sclerosis. Each was based on populations of Northern European origin ${ }^{1-4}$ and all four failed to identify major predisposing genes. Given that the power of a linkage genome screen is dependent upon the frequency of susceptibility alleles in the population studied and is thus expected to vary between populations, ${ }^{5}$ we reasoned that despite the failure of previous linkage screens, this approach was still worth pursuing in a population where the frequency of susceptibility alleles might be more favourable for historical reasons.

The prevalence of multiple sclerosis in Sardinia $(\approx 140$ per $100000)$ is much higher than in surrounding Mediterranean countries suggesting that the isolated growth of this population has concentrated susceptibility factors for the disease, making it an ideal place to screen for linkage. In order to utilise the special opportunity presented by the Sardinian

${ }^{*}$ Correspondence: MG Marrosu, Centro per la diagnosi e cura per la Sclerosi Multipla, Universita' di Cagliari, ASL8 Ospedale Binaghi, via Is Guaddazonis, 09100, Cagliari, Italy. Tel: 0039070 6092925;

Fax: 00390706092929 ; Email: gmarrosu@vaxca1.unica.it Received 27 November 2000; revised 18 April 2001; accepted 9 May 2001 population we have completed a whole genome screen for linkage in 49 Sardinian multiplex families (including 46 sibling pairs and three sibling trios) using 327 markers. This present study partially overlaps with the families and markers used in our previously reported partial genome screen, which involved 69 markers typed in 28 families. $^{6}$

\section{Materials and methods \\ Patients}

The relatives of index cases and their affected siblings were recruited by the Regional Center for Multiple Sclerosis in Cagliari (Southern Sardinia) and by the Neurology Department of the University of Sassari (Northern Sardinia). All patients had clinically definite multiple sclerosis according to the criteria of Poser. ${ }^{7}$ Magnetic resonance imaging demonstrated that all patients had white matter lesions consistent with demyelination, according to the criteria of Paty. ${ }^{8}$ A total of 49 families were studied in the linkage analysis: 46 sibling pairs and three sibling trios. Unaffected siblings $(n=54)$ and parents $(n=60)$ were included in the study, when available. An additional seven affected parent-child pairs were genotyped but these were only used in the search for linkage-disequilibrium. 


\section{Markers}

We screened the genome using the set of microsatellite markers developed by Reed et $a l^{9}$ together with HLA markers and additional microsatellites added to improve the coverage. ${ }^{10}$ In total, 327 markers (324 microsatellites together with HLA DRB1, DQA1 and DQB1) were used. The mean observed heterozygosity for the markers was $77 \%$ and their mean PIC was $75 \%$. The marker order and positions are indicated in Figure 1.
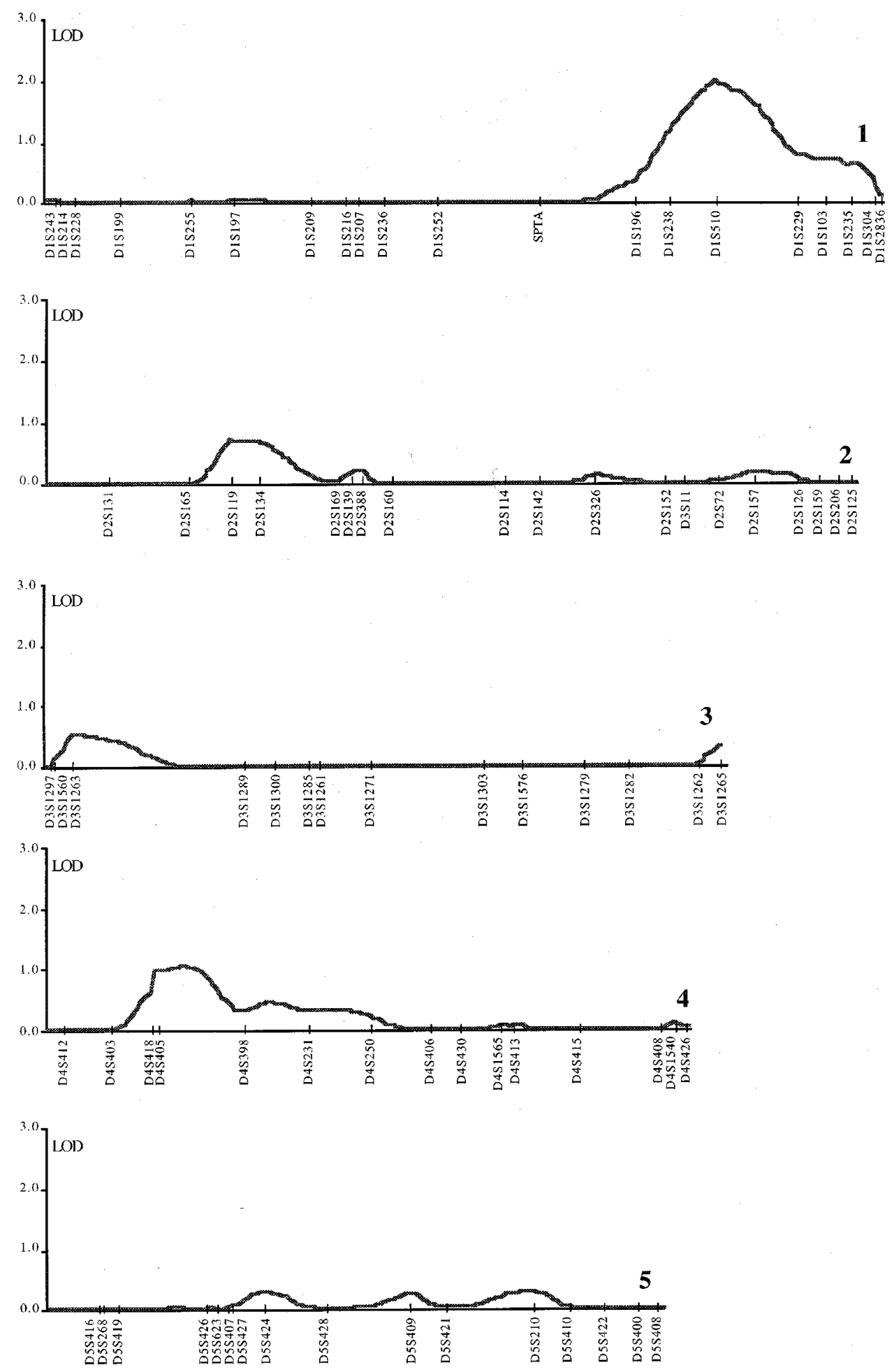
Figure 1 The lod score calculated by MAPMAKER-SIBS ${ }^{14}$ at each point along the genome is indicated, one graph for each chromosome. The length of each $\mathrm{x}$ axis is proportional to the genetic length of the corresponding chromosome and the map position of the markers is indicated by the tick marks. Marker names are placed as near as possible to their appropriate tick mark within the limits of clarity; tick marks for markers with less than $2 \mathrm{CM}$ separation have in some instances been

superimposed. The $y$-axis is scaled from 0 to 3.0 in each case.
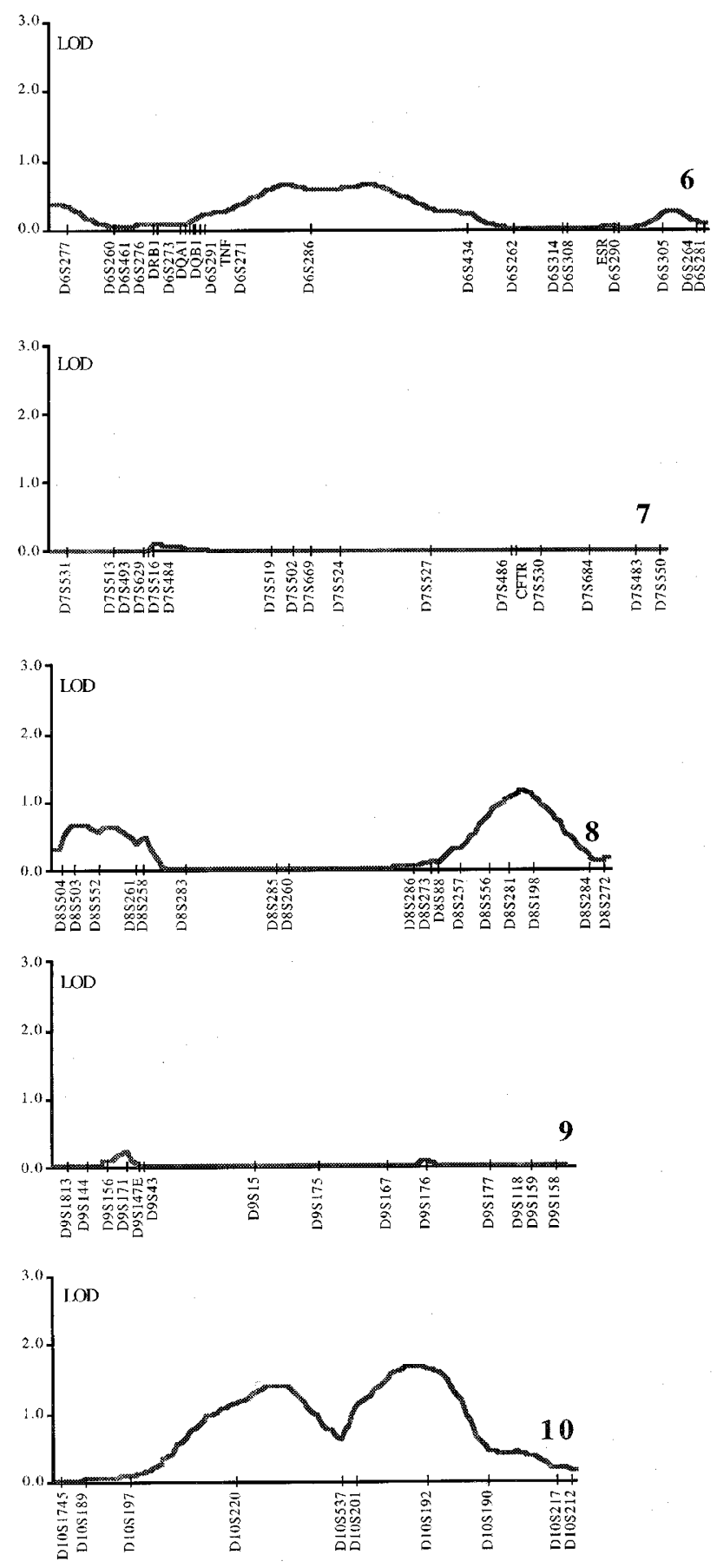
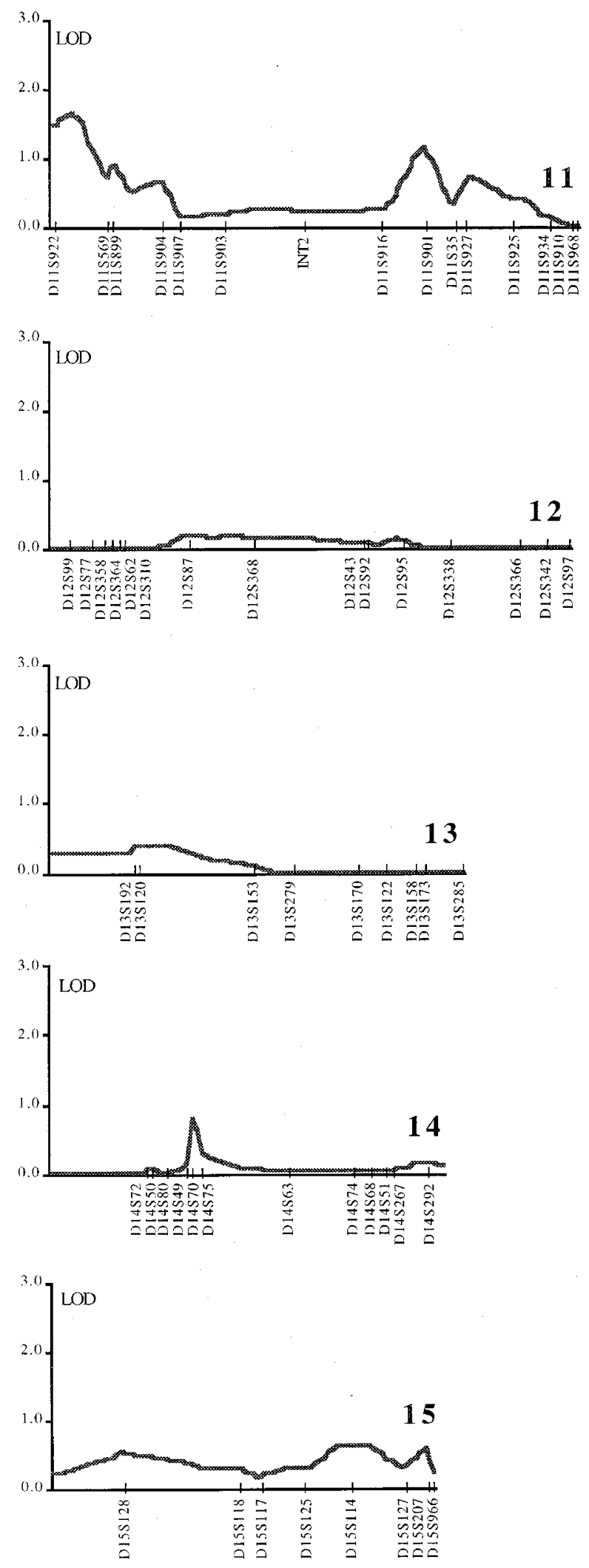

European Journal of Human Genetics 

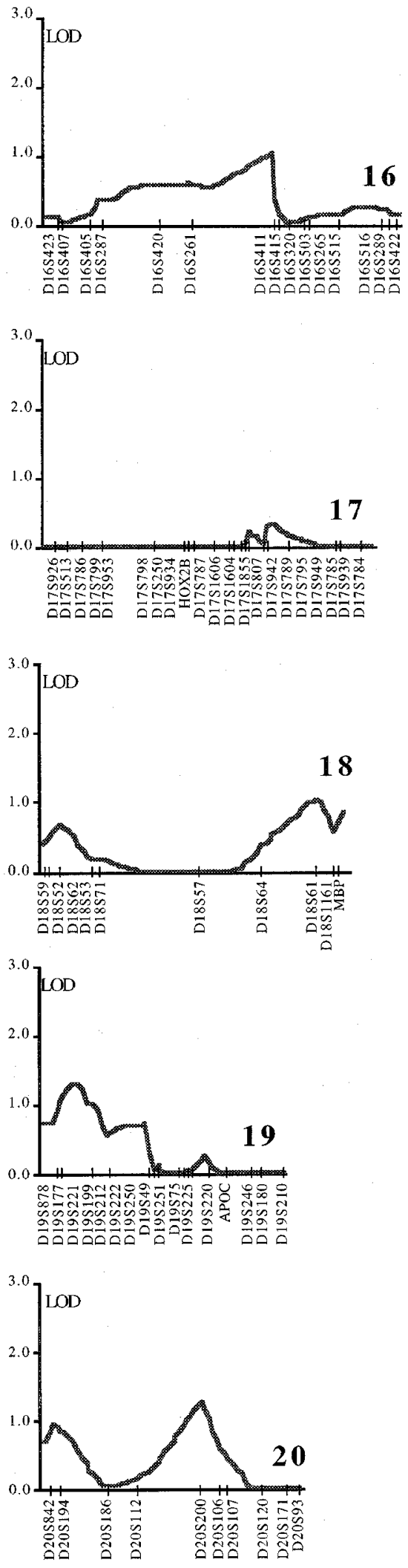
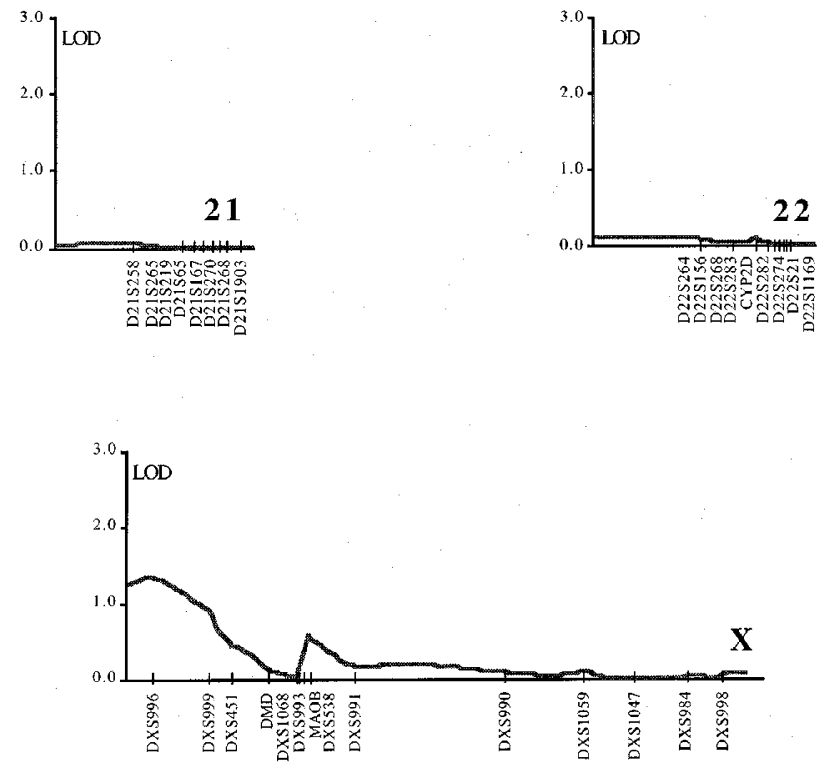

Map

The genetic position of each marker was established from the location database (LDB, http://cedar.genetics.soton.ac.uk/ public_html/). ${ }^{11}$ Microsatellite markers not included in this database were placed midway between their flanking markers according to Davies et al. ${ }^{12}$ Genetic distances between markers were calculated from their LDB locations and were adjusted to the nearest $\mathrm{cm}$. Where the separation was less than $0.5 \mathrm{~cm}$, these were rounded up to $1 \mathrm{~cm}$ (except for five markers in the region of HLA where spanning at $0.2 \mathrm{~cm}$ was used). This analysis was performed using the map version available on January 17th 2000.

\section{Genotyping}

In order to minimise the usage of genomic DNA, a primer extension pre-amplification (PEP) protocol was used to nonspecifically amplify each sample prior to genotyping. ${ }^{13}$ Polymerase chain reactions (PCR) were performed on Hybaid Omnigene and GRI Tetrad machines in $10 \mu \mathrm{l}$ reaction volumes using the procedure described elsewhere. ${ }^{9}$ For each microsatellite the forward primer was fluorescently labeled. In each case thermal cycling involved an initial touchdown phase over 10 cycles, followed by 20 cycles with $1 \mathrm{~min}$ denaturing at $94^{\circ} \mathrm{C}, 1 \mathrm{~min}$ annealing at $55^{\circ} \mathrm{C}$ and $45 \mathrm{~s}$ extension at $72^{\circ} \mathrm{C}$, and ending with a single extension step of $10 \mathrm{~min}$ at $72^{\circ} \mathrm{C}$. PCR products were combined in sets to allow semi-automated typing on Applied Biosystems 373A sequencing machines using GENESCAN and GENOTYPER software (Perkin Elmer). ${ }^{9}$

\section{Statistical methods}

Non parametrical linkage analysis Non parametrical linkage analysis was performed using the MAPMAKER/SIBS program. ${ }^{14}$ The maximum likelihood allele frequencies used 
in the linkage analysis were determined from the data using the SPLINK program version 1.09 (ftp://ftp.mrc-bsu.cam. ac.uk/pub/methodology/genetics/). In order to prevent the accidental inclusion of unrelated individuals or half sibs as apparent sib pairs, all pairs were checked with the SIBERROR program, ${ }^{15}$ and no errors were identified.

\section{Transmission disequilibrium testing}

Evidence for linkage disequilibrium was sought in the data from all 56 families using the TRANSMIT program version 2.5 (ftp://ftp.mrc-bsu.cam.ac.uk/pub/methodology/genetics/). The statistical significance of observed distortion in transmission ( $P$ values) was assessed using TRANSMIT's bootstrap function.

\section{Results}

The results of multipoint non-parametric linkage analysis on each chromosome are shown in Figure 1. In three regions (Chr 1q31, Chr 10q23 and Chr 11p15) the MLS exceeds 1.8, the threshold considered to represent suggestive linkage for a map of this density. ${ }^{16,17}$ The average information extraction across the genome was 51\%. Each of the microsatellite markers was also tested for evidence of transmission disequilibrium using the TRANSMIT program but no significant associations were seen after correcting for multiple testing.

\section{Discussion}

We have completed a genome wide screen for linkage in 49 Sardinian families with multiple sclerosis. Several regions of interest have been identified. In three regions: Chr 1q31, Chr $10 \mathrm{q} 23$ and Chr 11p15, the MLS exceeds the threshold suggestive of linkage for a map with this density. ${ }^{16,17}$

The chromosome 10 result is interesting as this region was also identified in the American-French genome screen. ${ }^{2}$ We also found another smaller peak (MLS 1.5) of potential linkage on $10 \mathrm{q} 11.21$ in the same region as $i d d m 10^{18}$ raising the possibility that a locus conferring susceptibility to autoimmunity in general might be encoded there. Our other peak of potential linkage at chr11p 15.5 coincides with iddm2, the insulin gene, ${ }^{12}$ while our third peak on Chr1q31 includes the PTPRC (protein-tyrosine phosphatase, receptortype C) gene which as recently been implicated in multiple sclerosis. $^{19}$

The association of class II HLA alleles with multiple sclerosis is well established but the effect of this locus on susceptibility is modest and therefore evidence for linkage in this region is expected to be limited, as in the previous genome screens. ${ }^{1,3,4}$ A recent study performed on Sardinian multiplex families estimates a symbol $\lambda$ s of only 1.2 for the HLA region, ${ }^{20}$ and it is therefore not surprising that we only found limited evidence for linkage in the HLA region. While not unexpected this finding suggests that other regions outside HLA contribute as much or more to disease susceptibility in Sardinian multiple sclerosis. The previous linkage genome screens in multiple sclerosis ${ }^{1-4}$ concentrated on individuals of northern European descent and clearly demonstrated that, at least in these populations, the individual effects of genes determining susceptibility to the disease are modest $(\lambda s \leqslant 2) .{ }^{21,22}$ Genetic isolates represent an exceptional resource in the identification of disease loci. Despite the high prevalence of multiple sclerosis in Sardinia, the at risk population is only 1600000 and therefore the total number of multiplex families available for study is limited (we estimate that at least $80 \%$ of the total number available have been included in this analysis). Our hypothesis was that, given the special nature of the Sardinian population, the frequency of susceptibility alleles might be more favourable and thereby enable linkage to be identified even in a small sample. ${ }^{5}$ The failure of linkage genome screens to identify with confidence regions of interest with respect to disease susceptibility both in mixed and isolated European populations, each having a high prevalence of multiple sclerosis, has implications for genetic analysis of complex traits. We feel future strategies should continue to include linkage analysis in multiplex families but recognise that other approaches will also be necessary. Association methods should be used to screen the whole genome and emerging regions of interest, despite the uncertain degree of linkage disequilibrium and larger genotyping effort. Meanwhile, continued scrutiny of the human genome map for intelligent and positional candidates may shorten the search for genes that determine susceptibility and influence the course of multiple sclerosis.

\section{Acknowledgments}

We thank the members of Professor Marrosu's laboratory for help in collecting and extracting the DNA samples from the Sardinian multiplex families and Julia Gray, Belinda Smillie and Mel Maranian for technical support during this study. The DNA collection has been supported by a grant from the Associazione Italian Sclerosi Multipla. We are grateful to the Wellcome Trust for financial support. F. Coraddu is supported by a Wellcome Trust grant and received a Sackler Award from the University of Cambridge.

\section{References}

1 Sawcer SJ, Jones HB, Feakes R et al: A genome screen in multiple sclerosis reveals susceptibility loci on chromosomes $6 \mathrm{p} 21$ and 17q22. Nat Genet 1996; 13: 464-468.

2 Haines JL, Ter-Minassian M, Bazyk A et al: A complete genomic screen for multiple sclerosis underscores a role for the major histocompatibility complex. Nat Genet 1996; 13: 469-471.

3 Ebers GC, Kukay K, Bulman DE et al: A full genome search in multiple sclerosis. Nat Genet 1996; 13: 472-476.

4 Kuokkanen S, Gschwend M, Rioux JD et al: Genomewide scan of multiple sclerosis in Finnish multiplex families. Am J Hum Genet 1997; 61: 1379-1387.

5 Risch N, Merikangas K: The future of genetic studies of complex human diseases. Science 1996; 273: 1516-1517. 
6 D'Alfonso S, Nistico L, Zavattari P et al: Linkage analysis of multiple sclerosis with candidate region markers in Sardinian and continental Italian families. Eur J Hum Genet 1999; 7: 377 385.

7 Poser CM, Paty DW, Scheinberg L et al: New diagnostic criteria for multiple sclerosis: guidelines for research protocols. Ann Neurol 1983; 13: $227-231$.

8 Paty D: Magnetic resonance in MS. Curr Opin Neurol 1993; 6: 202-208.

9 Reed PW, Davies JL, Copeman JB et al: Chromosome-specific microsatellite sets for fluorescence-based, semiautomated genome mapping. Nat Genet 1994; 7: 390-395.

10 Chataway J, Feakes R, Coraddu F et al: The genetics of multiple sclerosis: Principles, background and updated results of the United Kingdom systematic genome screen. Brain 1998; 121: $1869-1887$.

11 Collins A, Frezal J, Teague J, Morton NE: A metric map of humans:23,500 loci in 850 bands. Proc Natl Acad Sci USA 1996; 93: $14771-14775$

12 Davies JL, Kawaguchi Y, Bennett ST et al: A genome-wide search for human type 1 diabetes susceptibility genes. Nature 1994; 371: $130-136$.

13 Zhang L, Cui X, Schmitt K, Hubert R, Navidi W, Arnheim N: Whole genome amplification from a single cell: Implications for genetic analysis. Proc Natl Acad Sci USA 1992; 89: 5847 - 5851.
14 Kruglyak L, Lander ES: Complete multipoint sib-pair analysis of qualitative and quantitative traits. Am J Hum Genet 1995; 57: $439-454$.

15 Ehm M, Wagner M: A test statistic to detect errors in sib-pair relationships. Am J Hum Genet 1998; 62: 181-188.

16 Lander E, Kruglyak L: Genetic dissection of complex traits: guidelines for interpreting and reporting linkage results. Nat Genet 1995; 11: $241-247$.

17 Sawcer SJ, Jones HB, Judge D et al: Empirical genomewide significance levels established by whole genome simulations. Genet Epidemiol 1997; 14: 223-229.

18 Reed P, Cucca F, Jenkins S et al: Evidence for a type 1 diabetes susceptibility locus (IDDM10) on human chromosome 10p11q11. Hum Mol Genet 1997; 6: 1011 - 1016.

19 Jacobsen M, Schweer D, Ziegler A et al: A point mutation in PTPRC is associated with the development of multiple sclerosis. Nat Genet 2000; 26: $495-499$.

20 Marrosu M, Fadda E, Mancosu C, Lai M, Cocco E, Pugliatti M: The contribution of HLA to multiple sclerosis susceptibility in Sardinian affected sibling pairs. Ann Neurol 2000; 47: 411-412.

21 Sawcer SJ, Goodfellow PN, Compston DAS: The genetic analysis of multiple sclerosis. TIGs 1997; 13: 234-239.

22 Dyment DA, Sadovnick AD, Ebers GC: Genetics of multiple sclerosis. Hum Mol Genet 1997; 6: 1693-1698. 